\title{
High wall shear stress proximal to myocardial bridging and atherosclerosis: intracoronary ultrasound and pressure measurements
}

\author{
Junbo Ge, Raimund Erbel, Günter Görge, Michal Haude, Jürgen Meyer
}

\begin{abstract}
Background-Studies have shown that myocardial bridging may prevent coronary atherosclerosis and that the segment proximal to the bridge is often sclerosed. The underlying mechanism is still unknown.

Methods-Intracoronary ultrasound and pressure measurements were performed in a patient with myocardial bridging in the left anterior descending coronary artery. A 3.5 F, $20 \mathrm{MHz}$ probe was used to measure the change in cross sectional area of the lumen during the cardiac cycle. Intracoronary pressure was measured with a Double tip, end mounted pressure transducer system, the catheter having two pressure sensors located at the end of the catheter $3 \mathrm{~cm}$ apart. Intracoronary pressure was recorded as the catheter was slowly advanced and pulled back through the left anterior descending coronary artery.

Results-Systolic compression of the bridge segment was clearly visualised on ultrasonography and an eccentric plaque with calcium deposit was found in the segment proximal to the bridge. The pressure in the segment proximal to the bridge $(160 / 26 \mathrm{~mm} \mathrm{Hg})$ was higher than that of the proximal normal segment $(126 / 68 \mathrm{~mm} \mathrm{Hg})$. The pressure distal to the bridge was $68 / 30 \mathrm{~mm} \mathrm{Hg}$. A highly characteristic "sucking effect" was found in the bridge segment. The pressure in the bridge segment was 102/-40 mm $\mathrm{Hg}$.

Conclusion-The pressure in the segment proximal to the myocardial bridging was higher than aortic pressure. Disturbance of blood flow and high wall stress proximal to myocardial bridging was a main contributor to the development of atherosclerosis in the segment proximal to the bridge.
\end{abstract}

Keywords: atherosclerosis; ultrasonics; myocardium;

Myocardial bridging has aroused great interest as it might be the evidence of atherogenesis in coronary artery disease. Pathological and ultrasound studies show that atherosclerotic lesions seldom affect the bridge segment, ${ }^{1-4}$ although they are commonly found in the segment proximal to the bridge. ${ }^{3}$ Results from intracoronary Doppler ultrasonography show a characteristic blood flow pattern in the bridge segment, which suggests that atherosclerosis in the segment proximal to the bridge might be associated with high wall stress and subsequently intimal injury. ${ }^{3}$ We report simultaneous pressure measurement with a catheter pressure transducer system in a patient with myocardial bridging with the aim of elucidating the hypothesis of our previous study ${ }^{3}$ and examining in part the response to injury hypothesis. $^{5}$

\section{Patient and methods}

A 73 year old man attended for angiographic follow up four months after percutaneous transluminal coronary angioplasty of his left circumflex coronary artery. He had remained free of symptoms since the intervention. Left heart catheterisation showed an ejection fraction of $69 \%$. There was no regional wall motion abnormality. Coronary angiography showed a $40 \%$ reduction in the lumen of the left circumflex coronary artery in the segment that had been treated by angioplasty. A systolic "milking effect" was clearly shown.

\section{INTRACORONARY ULTRASONOGRAPHY}

We used a $3.5 \mathrm{~F}$ catheter and a $20 \mathrm{MHz}$ ultrasound transducer inside the catheter (Sonicath, Boston Scientific, Watertown, MA). The transducer rotated was mechanically within the catheter at $600-800 \mathrm{rpm}$ to provide cross sectional images on a diagnostic console (Diasonics, Milpitas, CA). An electrocardiogram was recorded simultaneously. The images were recorded on super VHS videotape for off line analysis.

MEASUREMENT OF INTRACORONARY PRESSURE We used a $3 \mathrm{~F}$ catheter with an end mounted pressure transducer system (Millar MIKROTIP, Millar instruments, Houston, TX). It had two pressure sensors, which were $3 \mathrm{~cm}$ apart at the end of the catheter. The type of sensor is diffused semiconductor with a pressure range of $-50 \mathrm{~mm} \mathrm{Hg}$ to $300 \mathrm{~mm} \mathrm{Hg}$. The sensitivity is $5 \mu \mathrm{V} / \mathrm{V} / \mathrm{mm} \mathrm{Hg}$. The catheter was connected to a transmitterreceiver (MIKRO-TIP, Millar) through the TC-510 control unit (MIKRO-TIP, Millar). The pressure was recorded using Electromingograph (Siemens, Erlangen, Germany).

\section{PROCEDURES}

The performance of intracoronary ultrasonography has been described in detail in the

\section{(Br Heart F 1995;73:462-465)} wall stress; intracoronary pressure \\ Cardiology, \\ R Erbel \\ $M$ Haude \\ University of Mainz, \\ Mainz, Germany
}


Figure 1

Electrocardiogram (top) recorded simultaneously during measurement of intracoronary pressure (bottom) with the proximal sensor located in the segment proximal to the myocardial bridge and the distal sensor located in the segment distal to the bridge (middle).

Figure 2

Electrocardiogram (top) recorded simultaneously during measurement of intracoronary pressure (bottom) with the proximal sensor in the proximal normal segment and the distal sensor in the bridge segment (middle). $A$ pressure gradient and $a$ characteristic "sucking effect" is clearly seen.
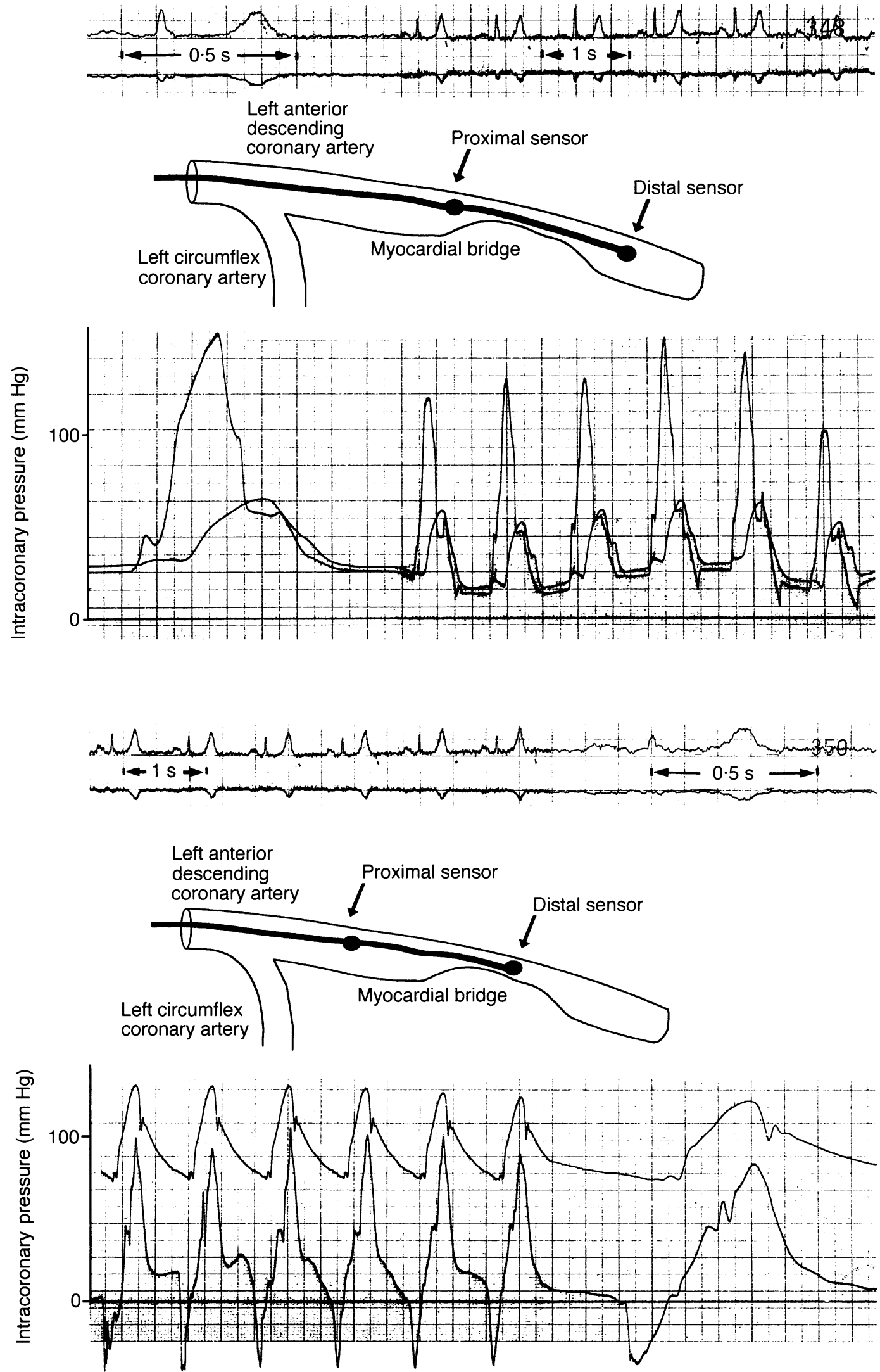

previous study. ${ }^{3}$ After the examination the guidewire was left in situ in the coronary artery. The pressure transducer system was then advanced over the wire to the left anterior descending coronary artery. The pressure was simultaneously recorded by slowly advancing and pulling back the catheter. The pulsatile variation in the bridge segment was analysed with a semiautomatic computer program. ${ }^{3}$

\section{Results}

INTRACORONARY ULTRASONOGRAPHY

Because of the dynamic stenosis of the artery, it was not possible to feed the ultrasound catheter through the whole bridging segment. An eccentric, systolic compression of the bridge segment was clearly visualised. The cross sectional area of the bridge segment was $2.5 \mathrm{~mm}^{2}$ in diastole and $1.7 \mathrm{~mm}^{2}$ in systole. No plaque was found in the bridge segment. 


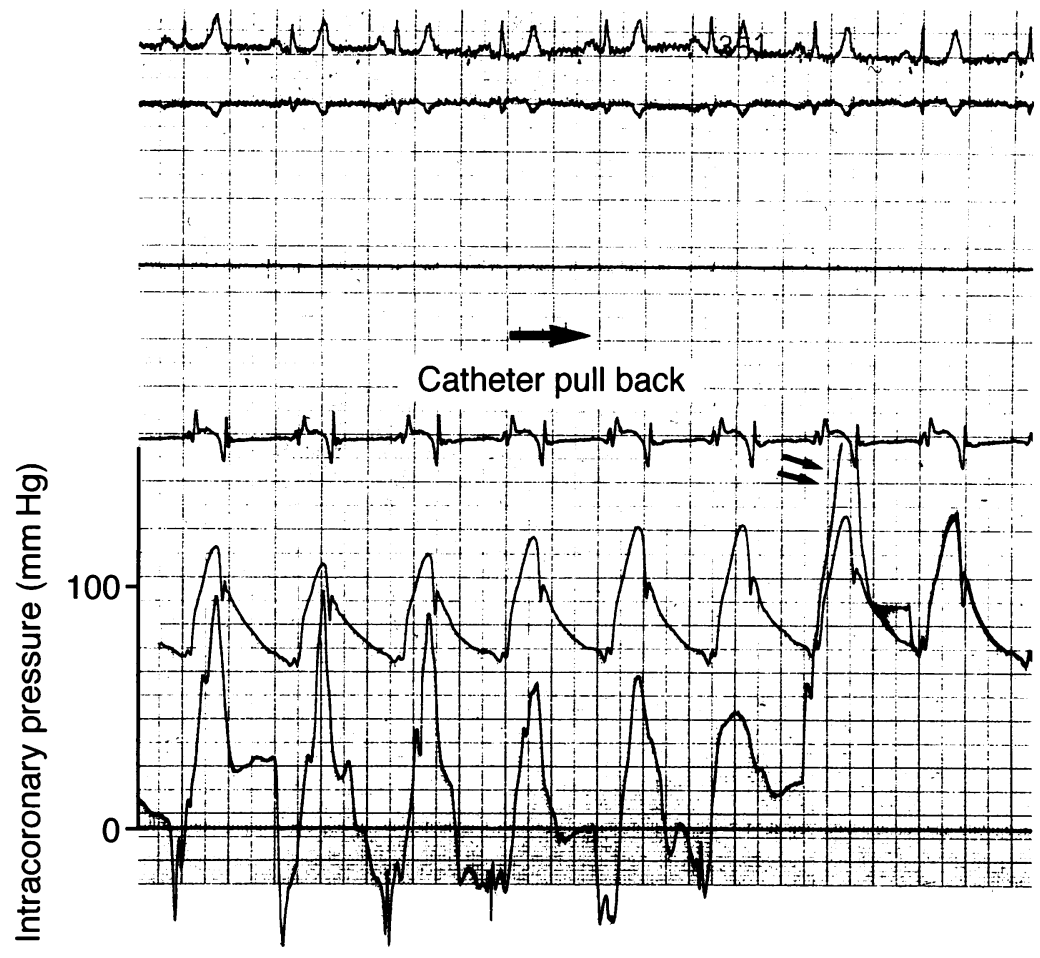

Figure 3 Electrocardiogram (top) and pressure pattern (bottom) when the pressure catheter was being pulled back. The pressure gradient disappeared when both pressure sensors were pulled back to the normal segment proximal to the myocardial bridge. When the distal pressure sensor passed through the segment just proximal to the myocardial bridge, the pressure overshot (double arrow).

An eccentric plaque with calcium deposits was found in the segment proximal to the bridge, which caused a maximal stenosis of $41 \%$.

\section{ANGIOGRAPHY}

A typical systolic "milking effect" was clearly seen on coronary angiography. No significant stenosis was detected in the segment proximal to the bridge segment.

PRESSURE MEASUREMENT

Figure 1 shows the pressure curve and the position of the pressure sensors. The distal sensor was located in the segment distal to the myocardial bridging, the proximal sensor just proximal to the bridge. The proximal pressure was $160 / 26 \mathrm{~mm} \mathrm{Hg}$. The pressure of the segment distal to the bridge was $68 / 30 \mathrm{~mm} \mathrm{Hg}$. A time shift of the pressure curve was clearly seen in the segment proximal to the bridge.

The pressure curve was continuously recorded as the pressure catheter was slowly pulled back. Figure 2 gives the pressure curve and the position of the pressure sensors. The proximal sensor was located in the normal segment and the distal sensor in the bridging segment. The proximal pressure was $126 / 68$ $\mathrm{mm} \mathrm{Hg}$. The pressure in the proximal bridging segment was $102 /-40 \mathrm{~mm} \mathrm{Hg}$. A characteristic pressure "sucking phenomenon" was seen (negative pressure in late diastole and a prominent pressure peak in late systole). When the pressure catheter was pulled back further, both sensors reached the normal segment and the pressure gradient disappeared.
Figure 3 shows that the distal sensor recorded a pressure spike (double arrow) when it passed the segment proximal to the bridge, which is the same as seen in figure 1 .

\section{Discussion}

To our knowledge, this is the first study to show that a high wall shear stress exists in the segment proximal to myocardial bridging. This explains to a large extent the high incidence of atherosclerosis in that segment ${ }^{3}$ and the effect of mural coronary artery in the "prevention" of coronary atherosclerosis. ${ }^{24}$ On the other hand, the "prevention" effect of atherosclerosis in the bridge segment might result from its better lymph drainage through myocardial compression. ${ }^{6}$

The pressure in the segment proximal to myocardial bridging $(160 / 26 \mathrm{~mm} \mathrm{Hg})$ was higher than that in the aorta $(126 / 68 \mathrm{~mm}$ $\mathrm{Hg}$ ). Maximal pressure in the segment proximal to the bridge was reached in mid-systole (pressure shift) and maximal pressure of the normal segment in late systole. The high pressure in the segment proximal to the bridge segment is most probably caused by the squeezing of the bridge during systole to produce retrograde flow against the anterograde flow. This phenomenon was observed in a previous study, in which blood flow velocity in the segment proximal to the bridge was negative. The shift in pressure is also probably caused by the retrograde flow produced by the compression of the bridge segment against the normal anterograde flow.

A characteristic pressure "sucking phenomenon" was observed in the bridge segment at end diastole (figure 2). This suggests that the myocardium dilates at end diastole, producing a negative pressure in the myocardial bridge. Although no pressure shift was noted, the pressure gradient between the normal segment and the bridge segment was significant. This indicates that the pressure and the corresponding wall shear stress are still lower than that of the normal segment, therefore preventing atherosclerosis in the vessel under the myocardial bridge.

The pathogenesis of atherosclerosis has been explored for decades and the process is still under investigation. Nevertheless, vascular injury and the resultant thrombus formation have been regarded as the key events in the origin and progression of atherosclerosis and in the pathogenesis of the acute coronary syndromes, ${ }^{7}$ whereas chronic minimal injury to the endothelium is caused mainly by a disturbance in the pattern of blood flow in certain parts of the arterial tree, such as bending points and areas near branching vessels. ${ }^{4-8}$ The disturbed blood flow pattern and increased pressure in the segment proximal to the bridge will inevitably lead to intimal injury and contribute to the development of atherosclerosis. In addition, high wall stress induced by high pressure proximal to the bridge may also be important in disrupting or fissuring plaque. ${ }^{910}$ Our study of intracoronary pressure measurement in a patient with myocardial 
bridging and another observation in patients with myocardial bridging ${ }^{3}$ strongly supports the previous pathological observation and the response to injury hypothesis. ${ }^{5}$ Our current observation also helps to explain the pattern of coronary blood flow in the bridge segment and the common finding of atherosclerosis in the segment proximal to the bridge. ${ }^{3}$

To date, only intracoronary ultrasonography is able accurately to evaluate the severity of the stenosis in coronary arteries because of the coronary arterial remodelling in the presence of atherosclerosis that compensates for narrowing of the lumen. ${ }^{11}$ Coronary angiography cannot be used to detect the early signs of atherosclerosis. $^{311}$ In our case we did not detect any significant narrowing of the lumen in the segment proximal to the bridge on coronary angiography. Intracoronary ultrasonography however, showed an eccentric stenosis of $41 \%$, which is undetectable on angiography. ${ }^{11}$

In conclusion, the pressure in the segment proximal to the myocardial bridging is higher than in the aorta. The common finding of atherosclerosis in the segment proximal to myocardial bridging is probably caused by the high wall stress and disturbance in blood flow in that segment.

1 Scholte M, Weis P, Prestele H. Die koronare Muskelbrücke des Ramus descendens anterior. Virchows Ach A Pathol Anat Histopathol 1977;375:23-36.

2 Lee SS, Wu TL. The role of the mural coronary artery in prevention of coronary atherosclerosis. Archives of Pathol 1972;93:32-5.

3 Ge J, Erbel R, Rupprecht HJ, Koch L, Kearney P, Görge G et al. Comparison of intravascular ultrasound and angiography in the assessment of myocardial bridging. Circulation 1994;89:1725-32.

4 Ferreira AG Jr, Trotter SE, König B Jr, Decourt LV, Fox K, Olsen EGJ. Myocardial bridges: morphological and Olsen EGJ. Myocardial bridges: morpholo

5 Ross R. The pathogenesis of atherosclerosis-an update. N Engl f Med 1986;314:488-500.

6 Caro CG, Lever MJ. The mass transport of the arterial wall: effect of mechanical stresses and vasoactive agents, including nitrates. $Z$ Kardiol 1983;72(suppl 3): 178-81.

7 Fuster V, Badimon L, Badimon JJ, Chesebro JH. The pathogenesis of coronary artery disease and the acute coronary syndromes. $N$ Engl $₹$ Med 1992;326:242-50.

8 Glagov V, Zarins C, Giddens DP, Ku DN. Hemodynamic and atherosclerosis: insights and perspective gained from studies of human arteries. Arch Pathol Lab Med 1988; 112:1018-31.

9 Richardson PD, Davies MJ, Born GVR. Influence of plaque configuration and stress distribution on fissuring of coronary atherosclerotic plaques. Lancet 1989;ii: 941-4.

10 Fuster V, Stein B, Ambrose JA, Badimon JJ, Chesebro JH. Atherosclerotic plaque rupture and thrombosis: evolving concepts. Circulation 1990;82:47-59.

11 Ge J, Erbel R, Zamorano J, Koch L, Kearney P, Görge G, Gerber $T$, et al. Coronary arterial remodeling in the atherosclerotic disease: an intravascular ultrasonic study in vivo. Coron Artery Dis 1993;4:981-6. 